\title{
Discovery of a Mid-infrared Echo from the TDE Candidate in the Nucleus of ULIRG F01004-2237
}

\author{
Liming Dou ${ }^{1,2}$, Tinggui Wang ${ }^{3}$, Lin Yan ${ }^{4,5}$, Ning Jiang ${ }^{3}$, Chenwei Yang ${ }^{3}$, Roc M. Cutri ${ }^{5}$, Amy Mainzer ${ }^{6}$, and Bo Peng ${ }^{3}$ \\ Center for Astrophysics, Guangzhou University, Guangzhou 510006, China; doulm@gzhu.edu.cn \\ 2 Astronomy Science and Technology Research Laboratory of Department of Education of Guangdong Province, Guangzhou 510006, China \\ ${ }^{3}$ CAS Key Laboratory for Researches in Galaxies and Cosmology, University of Sciences and Technology of China, Hefei, Anhui 230026, China \\ twang@ustc.edu.cn \\ ${ }^{4}$ Caltech Optical Observatories, Cahill Center for Astronomy and Astrophysics, California Institute of Technology, Pasadena, CA 91125, USA \\ ${ }^{5}$ IPAC, Mail Code 100-22, California Institute of Technology, 1200 E. California Boulevard, Pasadena, CA 91125, USA; lyan@ipac.caltech.edu \\ ${ }^{6}$ Jet Propulsion Laboratory, California Institute of Technology, Pasadena, CA 91109, USA \\ Received 2017 March 15; revised 2017 May 2; accepted 2017 May 3; published 2017 May 17
}

\begin{abstract}
We present the mid-infrared (MIR) light curves (LCs) of a tidal disruption event candidate in the center of a nearby ultraluminous infrared galaxy F01004-2237 using archival WISE and NEOWISE data from 2010 to 2016 . At the peak of the optical flare, F01004-2237 was IR quiescent. About three years later, its MIR fluxes have shown a steady increase, rising by 1.34 and $1.04 \mathrm{mag}$ in 3.4 and $4.6 \mu \mathrm{m}$ up to the end of 2016. The host-subtracted MIR peak luminosity is $2-3 \times 10^{44} \mathrm{erg} \mathrm{s}^{-1}$. We interpret the MIR LCs as an infrared echo, i.e., dust reprocessed emission of the optical flare. Fitting the MIR LCs using our dust model, we infer a dust torus of the size of a few parsecs at some inclined angle. The derived dust temperatures range from 590-850 K, and the warm dust mass is $\sim 7 M_{\odot}$. Such a large mass implies that the dust cannot be newly formed. We also derive the UV luminosity of 4-11 $\times 10^{44} \mathrm{erg} \mathrm{s}^{-1}$. The inferred total IR energy is $1-2 \times 10^{52} \mathrm{erg}$, suggesting a large dust covering factor. Finally, our dust model suggests that the long tail of the optical flare could be due to dust scattering.
\end{abstract}

Key words: black hole physics - galaxies: nuclei - infrared: galaxies

\section{Introduction}

When a star passes within a tidal radius $\left(r_{\mathrm{t}}=\left(M_{\mathrm{BH}} / M_{*}\right)^{1 / 3} r_{*}\right)$ of a supermassive black hole (SMBH), it will be torn apart by tidal forces. About half of the stellar material is on unbound orbits and escapes, and the other half is accreted by the central black hole, generating luminous flares in the soft X-ray, UV, and optical wavelengths, lasting a few months to years (Rees 1988, 1990; Hills 1975; Lidskii \& Ozernoi 1979). This is known as tidal disruption event (TDE). For a solar-type star and a non-rotating black hole $>10^{8} M_{\odot}$, the tidal radius will be inside the event horizon, then a TDE is not observable. So most TDEs occur in lower-mass SMBHs $\left(<10^{8} M_{\odot}\right)$. A dozen TDEs have been reported so far from transient surveys or serendipitous observations from X-ray to optical (see Komossa 2015 for a recent review).

TDEs serve as a powerful probe of the black hole accretion process itself. In addition, they offer a unique opportunity to study interstellar medium within a few parsecs surrounding quiescent SMBHs. The UV and soft X-ray flares from TDEs can ionize gas medium, and the subsequent recombination produces spectral signatures. These spectral features are detected as transient extreme coronal lines, variable [O III] lines, or broad transient $\mathrm{He}$ II and $\mathrm{H} \alpha$ lines (Komossa et al. 2008; Wang et al. 2011, 2012; Gezari et al. 2012; Yang et al. 2013; Arcavi et al. 2014; Holoien et al. 2016). When dust is present, UV/optical photons from TDEs are expected to be absorbed and re-emitted in the infrared. This dust emission is predicted to peak at 3-10 $\mu \mathrm{m}$ with an infrared luminosity of $10^{42-43} \mathrm{erg} \mathrm{s}^{-1}$ and last for a few years for a typical TDE (Lu et al. 2016). Such infrared emission has been detected for the first time recently in TDE candidates (Dou et al. 2016; Jiang et al. 2016; van Velzen et al. 2016) using archival data from Wide Field Infrared Survey Explorer (WISE) and Near Earth
Object Wide-field Infrared Survey Explorer Reactivation (NEOWISE-R) survey (Wright et al. 2010; Mainzer et al. 2014).

So far, most TDEs are found in normal post-starburst galaxies, whose star formation has been recently shut off (French et al. 2016). However, Tadhunter et al. (2017) recently reported the first case of a TDE candidate in a ULIRG F01004 -2237 at $z=0.1178$. They found a luminous optical flare with a $L_{\text {peak }} \sim 3 \times 10^{43} \mathrm{erg} \mathrm{s}^{-1}$ (peak date 2010 June 25) using the data from the Catalina Sky Survey (CSS; Drake et al. 2009). Following the optical flare, broad He II emission lines, were also detected in F01004-2237 (Tadhunter et al. 2017), which have been considered as the hallmarks of TDEs discovered from optical transient surveys (Arcavi et al. 2014; Blagorodnova et al. 2017). In addition, the estimated black hole mass is $\sim 2.5 \times 10^{7} M_{\odot}$ (Dasyra et al. 2006). Based on these characteristics, this optical flare was classified as a TDE candidate (Tadhunter et al. 2017).

This TDE candidate is unusual. First, the host galaxy has star formation rate $>100 M_{\odot} \mathrm{yr}^{-1}$ with massive young Wolf-Rayet stars in its compact nucleus (Surace et al. 1998). Both optical spectral line ratios and Spitzer MIR spectra suggest that it is a Seyfert 2 galaxy with a very dusty active galactic nucleus (AGN; Veilleux et al. 2009; Yuan et al. 2010). Second, the optical light curve (LC) has an unusually long decay timescale. Furthermore, three years after the peak, the LC did not fade back to its quiescent state. Instead, it levels off and is about 0.1 mag brighter. These unusual features cast many questions as to the true nature of this event. Could the optical flare be simply AGN variability? Is it possible that broad He II lines could be the result of variable accretion rates?

In this Letter, we report the MIR flare detected in F01004 -2237 three years after the optical peak date. The 3.4 and $4.6 \mu \mathrm{m}$ LCs are currently still rising. 


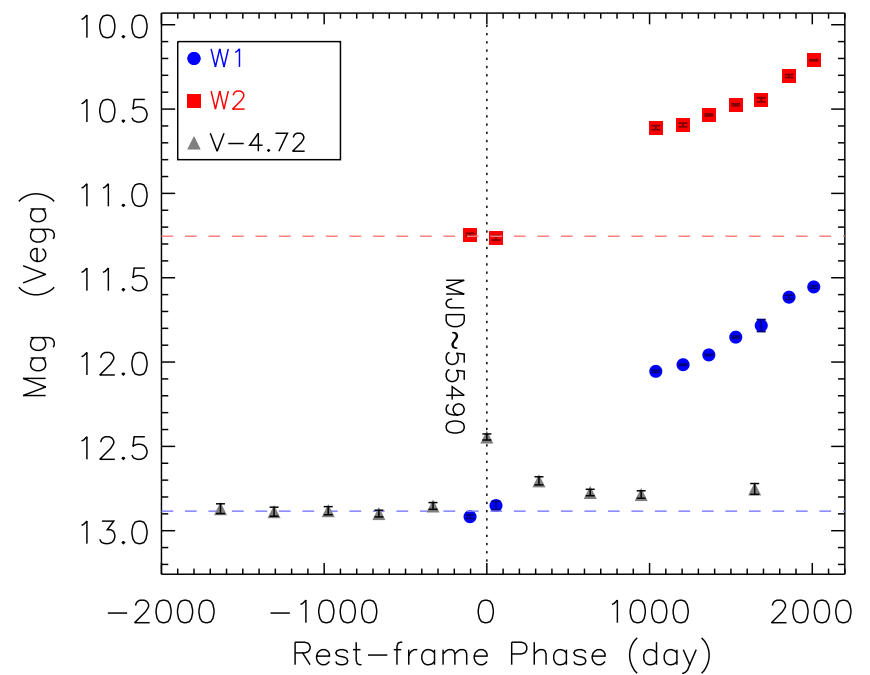

Figure 1. Light curves in V, W1, and W2 band of F01004-2237. The W1 and W2 band data are from WISE and NEOWISE-R, the V band data monitored by CSS are from Tadhunter et al. (2017). The rest-frame phase is relative to the date of the optical flare peak detected by the CSS (dotted line). The dashed lines are the average magnitudes in $\mathrm{W} 1$ and $\mathrm{W} 2$ bands of quiescent state. The $\mathrm{V}$ band light curve has been scaled down by 4.72 mag for clarity.

The Letter is organized as follows. The MIR LCs and results are described in Section 2. We discussed the results and concluded in Sections 3 and 4. Throughout this Letter, we adopt a $\Lambda$ CDM cosmology with $\Omega_{M}=0.3, \Omega_{\Lambda}=0.7$, and a Hubble constant of $H_{0}=70 \mathrm{~km} \mathrm{~s}^{-1} \mathrm{Mpc}^{-1}$. With the redshift of F01004-2237 $(z=0.1178)$, it results in a luminosity distance of $548.8 \mathrm{Mpc}$.

\section{Data Analysis and Result}

\subsection{Light Curves}

The primary WISE survey ended on 2011 February 1, when it was put into hibernation (Wright et al. 2010). The NEOWISE- $R$ survey began on 2013 December 13 (Mainzer et al. 2014). There was a $\sim 3$ year gap between WISE and NEOWISE-R. We extract a total of nine epochs of MIR photometry from the WISE/NEOWISE archive. There are 10-20 exposures in each epoch. We use only the best-quality single-frame images by selecting only detections with data quality flag "qual_frame" $>0$. This leaves 9-17 measurements for each epoch. F01004-2237 is a point-like source in all of the WISE images. Checking for potential contamination, we do not find any other sources within $10^{\prime \prime}$. We do not find shortterm variabilities within the individual epochs. We then average these fluxes to obtain a mean value at each epoch. We checked the MIR LCs of three other point sources close to our target, and no variability is detected in them. The W1 and W2 band LCs are shown in Figure 1. The V band LC from the CSS is also overplotted and scaled down by 4.72 mag for clarity (Tadhunter et al. 2017).

There are no significant variations in the $\mathrm{W} 1$ and $\mathrm{W} 2$ bands between the first two epochs, $\sim 120$ days before and $\sim 60$ days after the peak of optical flare detected by the CSS. At the first epoch, the WISE4-band photometry is $12.88 \pm 0.02$, $11.26 \pm 0.02,6.08 \pm 0.02$, and $3.10 \pm 0.02 \mathrm{mag}$ at $\mathrm{W} 1, \mathrm{~W} 2$, W3, and W4 bands, respectively, which is consistent with MIR fluxes derived from the broadband IR SED including the Spitzer IRS spectrum $(5-35 \mu \mathrm{m})$ taken in 2004 and the archival
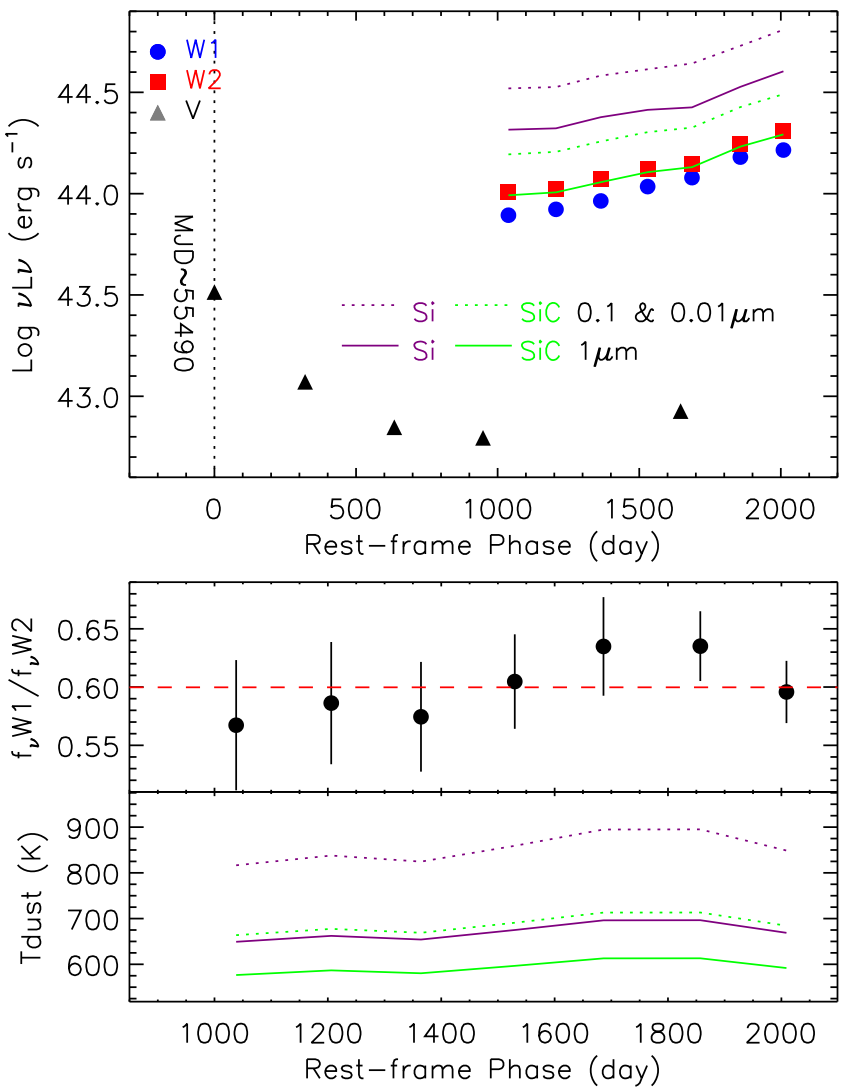

Figure 2. Upper panel: evolution of the host-subtracted luminosity in V, W1, and W2 bands of F01004-2237. The predicted 1-10 $\mu \mathrm{m}$ luminosities for Si (purple) and $\mathrm{SiC}$ (green) dust grains of varying sizes are also plotted. Middle panel: evolution of the ratio of host-subtracted flux between W1 and W2 bands. The red dashed line is the mean level. Lower panel: evolution of the dust temperature. The uncertainty of the dust temperature is typically $30-40 \mathrm{~K}$.

2MASS JHK photometry (Veilleux et al. 2009). This implies that the MIR emission is in a quiescent state during the first two epochs. The averaged brightness over these two epochs at 3.4 and $4.6 \mu \mathrm{m}$ is $12.88 \pm 0.04$ and $11.26 \pm 0.02 \mathrm{mag}$.

When F01004-2237 was re-observed during the NEOWISE$R$ survey $\sim 3$ years after the optical flare peak, it was 0.83 and 0.64 mag brighter in W1 and W2 bands, compared with the quiescent state. The source has been brightening steadily up to 2016 December 13 (the last epoch in Figure 1). Over the course of $\sim 6$ years after the optical flare, the W1 and W2 mag have brightened by 1.35 and $1.06 \mathrm{mag}$, respectively. After subtracting the quiescent flux, we show the MIR LCs in the upper panel of Figure 2. For comparison, we also show the LC of V band after subtraction of the constant component defined by pre-flare luminosity.

The host-subtracted, maximum MIR luminosity with the available data is $10^{44.2}$ and $10^{44.3} \mathrm{erg} \mathrm{s}^{-1}$ at 3.4 and $4.6 \mu \mathrm{m}$, respectively. This is a factor of 6 higher than the peak luminosity of the optical flare (host-subtracted). The strong silicate absorption in the pre-flare Spitzer MIR spectrum indicates this is a heavily dust obscured Seyfert 2 galaxy (Veilleux et al. 2009; Yuan et al. 2010). Therefore, the optical fluxes likely suffer from strong dust extinction.

\subsection{Dust Temperature and MIR Luminosity}

Because infrared fluxes are available only in the W1 and W2 bands during the NEOWISE-R survey, we rely on models to 

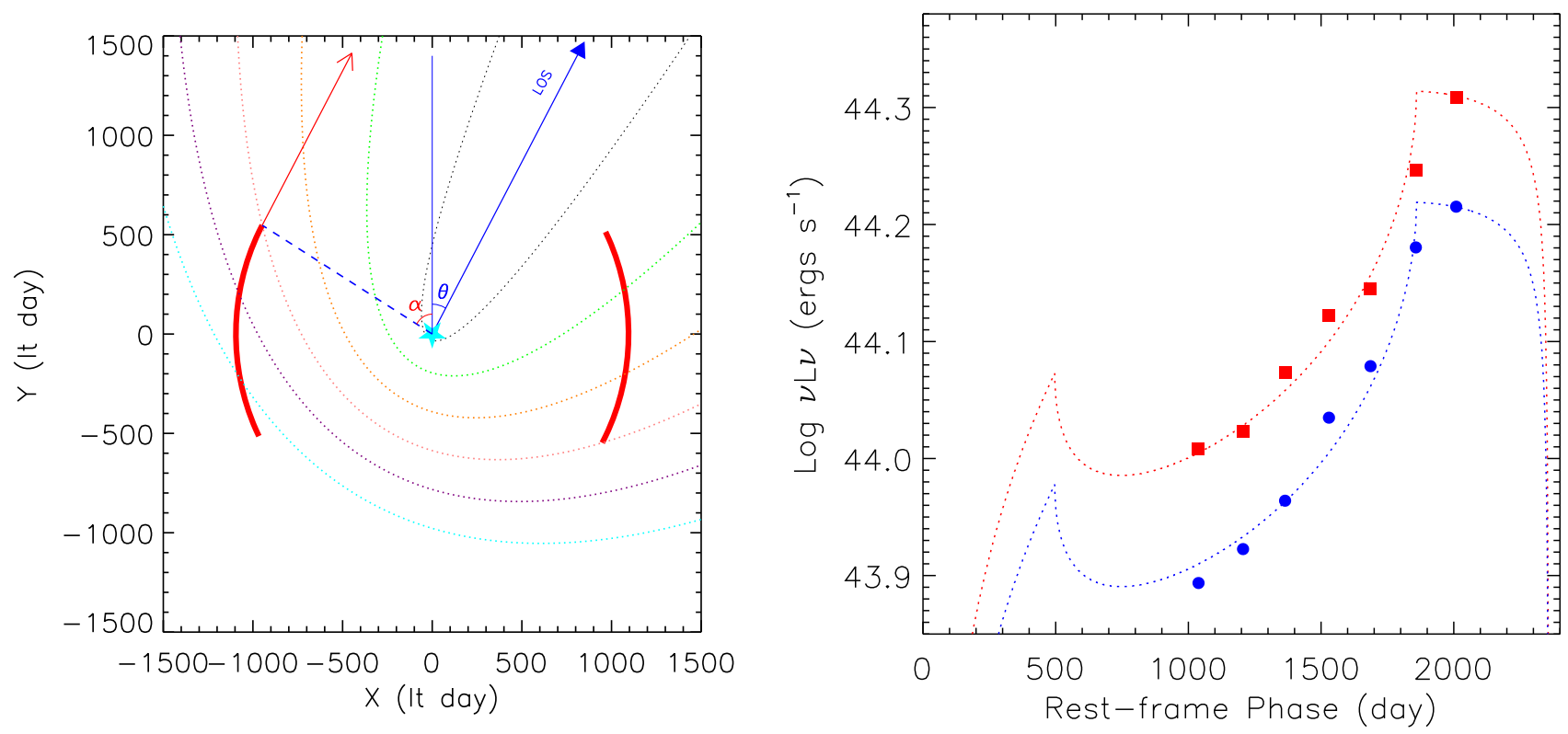

Figure 3. Left panel: schematic of a convex dusty ring (red bows) that absorbs UV photons and re-emits in the infrared and, simultaneously, scatter UV and optical photons into our line of sight. The dashed lines illustrate the iso-delay surface at lags of 60 days, 1,2,3,4, and 5 years. Right panel: example of theoretical light curves for the convex dusty ring model. We assume that infrared emission follows $I \propto \cos \Theta$, i.e., in optically thick case. The ring parameters are: $\theta=45^{\circ}, \alpha=70^{\circ}$, and $r=1.13 \mathrm{pc}$. The red (blue) dotted line is for W1 (W2) band.

estimate dust temperature. For simplicity, we consider a singletemperature dust consisting of either astronomical silicate $(\mathrm{Si})$ or silicon carbide $(\mathrm{SiC})$ with three different grain sizes, 0.01 , 0.1 , and $1 \mu \mathrm{m}\left(f_{\nu}=Q_{\text {abs }}(\nu) B\left(\nu, T_{d}\right)\right)$. The absorption efficiency $Q_{\mathrm{abs}}(\nu)$ for the dust is taken from Laor \& Draine (1993).

Applying these models to the host-subtracted W1 and W2 LCs, we estimate dust temperature and integral luminosity in $1-10 \mu \mathrm{m}$ at each epoch (see also Figure 2). The estimated dust temperatures appear to be almost constant as a function of time for a single grain size. This is due to the fact that the hostsubtracted $\mathrm{W} 1$ and $\mathrm{W} 2$ flux ratios are $\sim 0.6$, roughly constant over time. The derived $T_{d}$ ranges from 590 to $850 \mathrm{~K}$ with the assumed grain size of $0.01-1 \mu \mathrm{m}$, respectively. Finally, the luminosities derived from dust emission are in a narrow range of $(2-5) \times 10^{44} \mathrm{erg} \mathrm{s}^{-1}$, at the last epoch.

\section{Discussion}

\subsection{Dust Echo of a TDE?}

Extragalactic infrared variable sources can be categorized into two groups: non-thermal emission from relativistic jets such as blazars, and thermal dust emission as in radio-quiet AGNs and TDEs (Wang et al. 2017). Less than $10 \%$ of the known TDEs show jets, and the emission from a beamed jet often shows dramatic variability on timescale of hours to days (Jiang et al. 2012; Mangano et al. 2016) However, F01004 -2237 is radio quiet (Rodríguez Zaurín et al. 2013), during 3-6 years after the optical peak, its flux ratio between W1 and W2 band remains constant, and there are no significant short-term MIR variability. Thus, the observed infrared emission is unlikely to be non-thermal. For the remaining discussion, we consider only the thermal emission scenario. A UV flare, regardless of its origin as a changing-look AGN or a TDE, will cause a response in MIR emission if there is dust around the black hole at parsec distance scale. This is a so-called infrared echo. However, the MIR LC of F01004-2237 looks quite different from those of other TDEs/TDE candidates (Dou et al. 2016; Jiang et al. 2016; van Velzen et al. 2016). The question here is what conditions would produce an LC like this.

First, there is no significant MIR excess above the pre-flare level at 60 days after the optical flare. In other words, the UV/ optical photons from the flare had not been reprocessed by dust in 60 days. This implies that there must be very little dust reprocessing the UV flare along the line of sight within a radius of $30 \mathrm{lt}$-days from the optical flare source (Figure 3). ${ }^{7}$ Lack of dust within the radius of 30 lt-days at the peak of optical flare is expected because the dust sublimation radius is $>30$ lt-days when the peak luminosity of the optical flare exceeds ${ }^{8}$ $10^{43} \mathrm{erg} \mathrm{s}^{-1}$.

Second, our modeling of the data shows that the dust temperature remains roughly constant, suggesting that UV radiation intensity from the central source is also roughly the same at all epochs. Since the optical flare lasts a much shorter timescale than that of MIR LC, we can simplify and consider the optical flare as a pulse. Therefore, we infer that the dust distribution must be in a ring shape with equal distances to the central heating source. As shown in Figure 3, when the UV photons from the central source travel further out, they reach more and more surface areas of the dust torus, heating up more dust and producing rising MIR LCs. If this dusty ring is viewed at an intermediate angle, it can explain the lack of delay at less than 60 days and at the same time reproduce the continuous rise of the MIR flux. This was demonstrated by the similar doublehorn transfer function for an inclined ring calculated for broadline reverberation mapping study (Peterson \& Horne 2004). These horns are separated by $2 r \cos \theta / c$, where $r$ is the radius of the ring and $\theta$ is the inclination angle of the ring. Unfortunately, we do not have the early stage MIR LC, but

\footnotetext{
Strict speaking, there is little dust within the 60 day iso-lag surface. An isolag surface is a paraboloid with the UV source being its focus and the pericenter distance being $c \tau / 2$.

8 The dust sublimation radius is $R_{\text {sub }}=0.47\left(6 \nu L_{\nu}(V) /\left(10^{46} \mathrm{erg} \mathrm{s}^{-1}\right)\right)^{1 / 2} \mathrm{pc}$ (Kishimoto et al. 2007; Koshida et al. 2014).
} 
one prediction of the model is that MIR flux will turn over sharply after a certain time (see the right panel of Figure 3). This can be tested with future MIR observations. The radius of the ring is at least $3 \mathrm{lt}-\mathrm{yr}$, but not much larger, in order to match the LC.

Another interesting inference from the dust reprocessing scenario is the prediction of a dust echo signature (reflection component) in both the optical and UV bands. If dust composition and grain size are uniform over the region, we expect that the scattered optical flux is roughly proportional to that of reprocessed infrared light. The fraction of scattered light depends on the albedo of the dust, which is a strong function of grain size. Grains of size $>0.1 \mu \mathrm{m}$ will give strong reflection light up to $1 \mu \mathrm{m}$. With a reasonable albedo of dust in $\mathrm{V}$ band ( 0.5; Laor \& Draine 1993) and optical to UV light ratio of incident continuum $(\sim 0.2)$, scattered optical flux would be $\sim 10 \%$ of the infrared flux. This is sufficient to explain the puzzling up-turn in the optical LC several years after the optical flare.

\subsection{The UV Luminosity and Lower Limits to the Total Energy Release during the Flare}

Dust grains can be considered as bolometers due to large absorption cross-section in UV. Their temperature is determined by the incident UV flux. Its UV luminosity can be inferred from dust temperature and distance from the UV source. As discussed in the last section, the heated dust is likely in a convex surface with a distance to the UV source of at least 3 lt-yr. Using the formulae (1-3) in Dou et al. (2016) and adopting $Q_{\text {abs }}$ from Laor \& Draine (1993), one can estimate the UV luminosity.

For Si grains, the UV luminosity calculated from the best-fit convex dusty ring model is $(0.06,0.6$ and 4.6$) \times 10^{44} \mathrm{erg} \mathrm{s}^{-1}$ for grain size of $0.01,0.1$, and $1 \mu \mathrm{m}$, respectively. The value will be 1.4-2.2 times larger for $\mathrm{SiC}$ grains. The IR spectrum observed at any time is actually the sum of the emission components from where the dust ring intersects different iso-delay surfaces. Different areas are illuminated with different levels of UV luminosity and therefore have a range of equilibrium temperatures. Consequently, the single-temperature model described above underestimates the peak UV luminosity by a factor of 2-3. MIR spectra of the flare would allow us to constrain the dust properties. This should lead to a better estimate of the UV luminosity. Then, we will consider another way to estimate the integrated UV luminosity.

We calculate the total energy emitted in the infrared flare by integrating the host-subtracted LC. During the NEOWISE-R survey, F01004-2237 has emitted a total IR energy of about $1.3-2.0 \times 10^{52} \mathrm{erg}$. The dust covering factor is about 0.34 , inferred from the opening angle $\left(\alpha=70^{\circ}\right)$ of the best-fit convex dusty ring model. If the total IR energy comes from the heated optical/UV photons that released during the optical/UV flare, the total optical/UV energy should be at least $4 \times 10^{52} \mathrm{erg}$. This is about three times the energy released by a tidal disruption of a $0.3 M_{\odot}$ star proposed by Tadhunter et al. (2017), assuming half of the debris is accreted with a typical radiation efficiency of $\eta=0.1$. Given the fact that the early rising part of the LC was not observed, and the source is still brightening in MIR, the above energy is a lower limit. This suggests that the disrupted star is likely to be more massive or that the radiation efficiency is higher than 0.1 . If the timescale of the optical/UV flare is one year (Tadhunter et al. 2017), the integrated UV luminosity should be at least $1.2 \times 10^{45} \mathrm{erg} \mathrm{s}^{-1}$. Roughly, this value is consistent with the one estimated from the dust temperature and dust ring model for the largest grains considered.

We estimate the heated dust mass at each epoch using the formula (8-9) in Dou et al. (2016), adopting $\rho=2.5 \mathrm{~g} \mathrm{~cm}^{-3}$ for $\mathrm{Si}$. The heated dust mass is $\sim 5 M_{\odot}$ at the last epoch. In our model, the dust was emitting MIR photons for the duration of the optical flare and then cools down. So the lower limit to the total amount of dust is $\sim 7 M_{\odot}$, if it is assumed the optical flare timescale is one year. Such a large dust mass within a parsec radius of the SMBH implies that these dust grains cannot be newly formed. They are likely already there from the dusty torus of the Seyfert 2 nucleus.

\section{Conclusions}

We report the discovery of a strong infrared echo of the optical flare from the TDE candidate in ULIRG F01004-2237 using archival WISE and NEOWISE data. The object is quiescent in the MIR during the peak of the optical flare in 2010. However, three years after the optical peak, we find a steady rise in MIR emission, with fluxes increasing by a factor of 3.4 and 2.6 at $3.4 \mu \mathrm{m}$ and $4.6 \mu \mathrm{m}$, respectively. The peak MIR luminosities are $1.6 \times 10^{44}$ and $2.0 \times 10^{44} \mathrm{erg} \mathrm{s}^{-1}$ in the $\mathrm{W} 1$ and W2 bands, respectively, which are about $0.7-0.8 \mathrm{dex}$ higher than the observed peak flare luminosity in the $\mathrm{V}$ band. Despite the infrared luminosity continuously rising by a factor of 2 in the past three years, our model suggests that the temperature remained roughly constant at $590-850 \mathrm{~K}$. We estimate MIR energy radiated between 2013 and 2016 to be $(1.3-2.0) \times 10^{52}$ erg. We interpret the variable infrared emission in the context of thermal dust emission in response to the $\mathrm{UV} /$ optical flare, presumably caused by a TDE. A thick dusty ring with a radius of about $1 \mathrm{pc}$, mass $>7 M_{\odot}$, and grain size larger than $0.1 \mu \mathrm{m}$ around the black hole at an intermediate inclination angle may be able to reproduce all characteristics in the observed MIR LC. The model predicts a sharp decrease of the MIR flux not too far in the future, which can be verified by future photometric monitoring, and an optical component due to dust scattering of the primary UV/optical flare at a level $10 \%$ of the infrared luminosity, which may explain the very flat optical LC at the very late stage, and can be tested with polarization observations.

We are grateful to the anonymous referee for comments that have improved the quality of this Letter. We thank Wenbin Lu for helpful discussions and comments. This research is supported by the National Basic Research Program of China (grant No. 2015CB857005), NSFC (NSFC-11233002, NSFC11421303, NSFC-11603021), Joint Research Fund in Astronomy (U1431229, U1531245) under cooperative agreement between the NSFC and the CAS and the Fundamental Research Funds for the Central Universities. This research makes use of data products from the Wide-field Infrared Survey Explorer (WISE) and the Near-earth Object Wide-field Infrared Survey Explorer (NEOWISE). WISE is a joint project of the University of California, Los Angeles, and the Jet Propulsion Laboratory/ California Institute of Technology; NEOWISE is a project of the Jet Propulsion Laboratory/California Institute of Technology. WISE and NEOWISE are funded by the National Aeronautics and Space Administration. 


\section{References}

Arcavi, I., Gal-Yam, A., Sullivan, M., et al. 2014, ApJ, 793, 38

Blagorodnova, N., Gezari, S., Hung, T., et al. 2017, ApJ, submitted (arXiv:1703.00965)

Dasyra, K. M., Tacconi, L. J., Davies, R. I., et al. 2006, ApJ, 638, 745

Dou, L., Wang, T., Jiang, N., et al. 2016, ApJ, 832, 188

Drake, A. J., Djorgovski, S. G., Mahabal, A., et al. 2009, ApJ, 696, 870

French, K. D., Arcavi, I., \& Zabludoff, A. 2016, ApJL, 818, L21

Gezari, S., Chornock, R., Rest, A., et al. 2012, Natur, 485, 217

Hills, J. G. 1975, Natur, 254, 295

Holoien, T. W.-S., Kochanek, C. S., Prieto, J. L., et al. 2016, MNRAS, 455,2918

Jiang, N., Dou, L., Wang, T., et al. 2016, ApJL, 828, L14

Jiang, N., Zhou, H.-Y., Ho, L. C., et al. 2012, ApJL, 759, L31

Kishimoto, M., Hönig, S. F., Beckert, T., \& Weigelt, G. 2007, A\&A, 476, 713

Komossa, S. 2015, JHEAp, 7, 148

Komossa, S., Zhou, H., Wang, T., et al. 2008, ApJL, 678, L13

Koshida, S., Minezaki, T., Yoshii, Y., et al. 2014, ApJ, 788, 159

Laor, A., \& Draine, B. T. 1993, ApJ, 402, 441

Lidskii, V. V., \& Ozernoi, L. M. 1979, PAZh, 5, 28

Lu, W., Kumar, P., \& Evans, N. J. 2016, MNRAS, 458, 575
Mainzer, A., Bauer, J., Cutri, R. M., et al. 2014, ApJ, 792, 30

Mangano, V., Burrows, D. N., Sbarufatti, B., \& Cannizzo, J. K. 2016, ApJ, 817,103

Peterson, B. M., \& Horne, K. 2004, AN, 325, 248

Rees, M. J. 1988, Natur, 333, 523

Rees, M. J. 1990, Sci, 247, 817

Rodríguez Zaurín, J., Tadhunter, C. N., Rose, M., \& Holt, J. 2013, MNRAS, 432, 138

Sanders, D. B., \& Mirabel, I. F. 1996, ARA\&A, 34, 749

Surace, J. A., Sanders, D. B., Vacca, W. D., Veilleux, S., \& Mazzarella, J. M. 1998, ApJ, 492, 116

Tadhunter, C., Spence, R., Rose, M., Mullaney, J., \& Crowther, P. 2017, NatAs, 1, 0061

van Velzen, S., Mendez, A. J., Krolik, J. H., \& Gorjian, V. 2016, ApJ, 829, 19

Veilleux, S., Rupke, D. S. N., Kim, D.-C., et al. 2009, ApJS, 182, 628

Wang, T.-G., Yan, L., Dou, L., et al. 2017, MNRAS, submitted

Wang, T.-G., Zhou, H.-Y., Komossa, S., et al. 2012, ApJ, 749, 115

Wang, T.-G., Zhou, H.-Y., Wang, L.-F., Lu, H.-L., \& Xu, D. 2011, ApJ, 740,85

Wright, E. L., Eisenhardt, P. R. M., Mainzer, A. K., et al. 2010, AJ, 140, 1868

Yang, C.-W., Wang, T.-G., Ferland, G., et al. 2013, ApJ, 774, 46

Yuan, T.-T., Kewley, L. J., \& Sanders, D. B. 2010, ApJ, 709, 884 